\title{
Transition from Non-Fickian to Fickian longitudinal transport through 3-D rough fractures:
}

\section{Scale-(in)sensitivity and roughness dependence}

(1)

\author{
Lichun Wang ${ }^{1,2}$ and M. Bayani Cardenas ${ }^{1}$
}

${ }^{1}$ Department of Geological Sciences, University of Texas at Austin, Austin, Texas, USA;

${ }^{2}$ corresponding author: wanglichun@utexas.edu

\section{Abstract}

Understanding transport in rough fractures from non-Fickian to Fickian regimes and the prediction of non-Fickian transport is critical for the development of new transport theories and many practical applications. Through computational experiments that fall within the macrodispersion regime, we first simulated and analyzed solute transport through synthetic rough fractures with stationary geometrical properties (i.e., fracture roughness $\sigma_{b} /\langle b\rangle$ and correlation length $\lambda$, where $b$ refers to aperture with its standard deviation $\sigma_{b}$ and arithmetic mean $\langle b>$ ) across increasing fracture longitudinal transport domain length $L$, with $L / \lambda$ ranging from 2.5 to 50 . The results were used to determine how solute transport behavior evolves with increasing scale in the longitudinal direction. Moreover, a set of correlated fractures with aperture fields following normal and log-normal distributions was created to further identify and quantify the dependence of non-Fickian transport on roughness. We found that although persistent intermittent velocity structures were present, the breakthrough curves (BTCS) and residence time distributions showed diminishing early arrival and tailing, features of nonFickian transport, with increasing longitudinal $L / \lambda$, ultimately converging to a Fickian transport regime given $\sigma_{b} /<b>$ remained constant. Inverse analysis of the experimental BTCs with the 
22 advection-dispersion equation (ADE) model showed that the dispersion coefficient $(D)$ was non-

23 trivially scale-dependent. Simulation results for rough fractures with varying $\sigma_{b} /<b>$ and

$24 L / \lambda$ indicated that the ratio of fluid velocity to transport velocity fitted to the ADE model

25 depends on $\sigma_{b} /\langle b\rangle$ and $L / \lambda$. The continuous time random walk (CTRW) performed much better

26 across all transport scales, and resulted in scale-insensitive fitted parameters, i.e., $\beta$ in the

27 memory function. The fitted $\beta$ is proportional to $\sigma_{b} /\langle b\rangle$ but is insensitive to $L / \lambda$. Therefore, bulk

28 longitudinal solute transport across the pre-asymptotic and asymptotic regimes can be

29 estimated based on the CTRW model parameterized by measurable fracture physical properties.

\section{Introduction}

Solute transport through fractured media is subject to complex physical and chemical processes, characterized by preferential flow in fractures and slow/stagnant flow in the porous matrix or eddies, flow and solute exchange between the fracture and matrix (Roubinet et al., 2012), and fluid-rock interactions (Mukhopadhyay et al., 2013). The broad variations of velocity and chemical reactions consequently lead to non-Fickian transport across all scales of fractured media (Berkowitz, 2002). Unfortunately, accurate prediction of complex non-Fickian transport processes remains a challenge due to limitations in detailed description of single fractures, let alone discrete fracture networks and dual-porosity media (Neuman, 2005). As a result, numerous studies have focused on non-Fickian transport through single fractures (Bauget and

41 Fourar, 2008; James et al., 2005; James and Chrysikopoulos, 2000; Nowamooz et al., 2013;

42 Wang and Cardenas, 2014). 
44 heterogeneity in the flow field. First, the transport observation occurs at spatial and temporal scales that are too short or small, and therefore the solute fails to experience or sample the complete heterogeneity of the medium after where or when transport reaches the Fickian (Taylor) regime (e.g., Bolster et al., 2014; Bouquain et al., 2012; Koch and Brady, 1987;

Majdalani et al., 2015). Second, incomplete local mixing resulting from a complex flow field takes place (Koch and Brady, 1987), including for example a growing eddy (Cardenas et al., 2007; Cardenas et al., 2009; Sund et al., 2015). Even though the effect of heterogeneity (e.g., variance and correlation length) on the flow field has been documented extensively for decades, recent studies continue to quantify the impact of micro-scale heterogeneity on non-Fickian transport (Aubeneau et al., 2014; Heidari and Li, 2014; Kang et al., 2014).

Numerous studies have shown that fracture roughness (another indicator of heterogeneity for fractures) has a remarkable impact on the non-Fickian transport through single fractures (e.g., Bauget and Fourar, 2008; Cardenas et al., 2007; Keller et al., 1999). Fracture roughness typically refers to the roughness of fracture surfaces (Brown, 1987; Candela et al., 2012), but in this study, we define roughness $\left(\sigma_{b} /\langle b\rangle\right)$ as the variation of aperture field, where $\sigma_{b}$ and $\langle b\rangle$ are standard deviation and arithmetic mean of aperture $b$, respectively. The non-Fickian transport behavior can be approximated based on physical properties $\left(\sigma_{b} /\langle b\rangle\right)$ of two-dimensional (2-D) rough fractures following our previous study (Wang and Cardenas, 2014). However, transport through 2-D fractures does not allow for channelization which could occur in 3-D rough fractures, thus adding a mechanism for non-Fickian behavior. Therefore, the information needed for predicting non-Fickian transport through three-dimensional (3-D) rough fractures remains mostly unknown, particularly with increasing fracture scales. 
Although simulations of flow and transport through simple pores at increasing pore

67 scales have shown that the transport transitions from non-Fickian to Fickian regimes (Cardenas, 2009; Zhang and Lv, 2007), the effect of scale on non-Fickian transport through fractures needs

69 to be further studied in terms of identifying transition time and length scales, such that transport transition can be properly predicted (Bolster et al., 2014). Moreover, the determination of transition scales can help in the design of observation networks for experimental sampling or even field monitoring (Majdalani et al., 2015). To capture transport behavior, the classical advection-dispersion equation (ADE) with constant velocity and dispersion coefficient $D$ is widely used, even though its shortcomings for capturing non-Fickian behavior are widely recognized. Therefore a suite of nonlocal representations of advective and dispersive solute transport has emerged that more accurately

77 describes non-Fickian transport (Bauget and Fourar, 2008; Berkowitz et al., 2006; Neuman and

78 Tartakovsky, 2009). The continuous time random walk (CTRW) framework is one such approach

79 for modeling non-Fickian transport which has been applied extensively to fractures and fracture networks (Berkowitz et al., 2006; Cortis et al., 2004; Dentz et al., 2004; Le Borgne et al., 2008).

81 Moreover, the inclusion of the truncated power law (TPL) in time within the CTRW framework

82 led to improved agreement between theoretical breakthrough curves and observations for 83 systems with a finite scale (Cortis and Berkowitz, 2004). However, although the theoretical 84 prediction of transport across non-Fickian to Fickian transition is possible by using the CTRW-

85 TPL, there are few physical and even computational experiments that quantify such a

86 transitional transport phenomenon across increasing scales. Only a few studies directly assess

87 and directly connect the parameters of the CTRW-TPL to measurable physical properties 
(Mclnnis et al., 2014; Wang and Cardenas, 2014).

The CTRW-TPL, whose ability to represent macroscopic non-Fickian transport has been verified several times (Berkowitz, 2002; Berkowitz et al., 2006; Dentz et al., 2004; Kang et al., 2011), assumes that the temporal and spatial transitions are decoupled. This decoupled partial differential equation form of CTRW performs well regardless of whether the velocity field is correlated or not, as long as the spatial transitions are compact (Berkowitz and Scher, 2010).

Recent studies integrated information about velocity correlation via a spatial Markov method into the classic CTRW; this framework is sometimes called correlated CTRW (Bolster et al., 2014; Kang et al., 2014; Kang et al., 2011; Le Borgne et al., 2008). The correlated CTRW, for some cases with appropriate discretization of transition path, can accurately capture the early arrival and long tailing features (de Anna et al., 2013; Kang et al., 2011; Le Borgne et al., 2008). However, the correlated CTRW requires prior knowledge of particle distribution, which is normally unknown a priori, thus limiting its practical application. Therefore, instead of using the correlated CTRW, we investigated whether the classic CTRW-TPL framework is able to predict solute transport transitioning from non-Fickian to Fickian when velocity field is correlated; this was achieved by identifying an appropriate truncated time and other relevant parameters, and then relating the model parameters to the physical properties of 3-D rough fractures. We also compared the analysis with the CTRW-TPL framework to more conventional inverse modeling with the ADE.

In this study, we first investigated solute transport through single rough-walled fractures with increasing scales (fracture length) in order to quantify the effect of scale in potentially transforming transport from non-Fickian to Fickian regimes, and to further assess 
110 the usefulness and appropriateness of the CTRW-TPL framework for prediction across

111 transitional transport phenomenon but confined within the macrodisperison regime (Detwiler

112 et al., 2000). Then, we attempted to establish a relationship between the parameter $\beta$ in the

113 CTRW-TPL model and physical properties of 3-D rough fractures by changing fracture roughness

114 and correlation length; $\beta$ represents the nature of the transport, i.e., the degree of deviation

115 from Fickian transport, and encapsulates the degree of heterogeneity. We addressed the

116 following questions: How do the ADE and CTRW parameters change with fracture scale across,

117 presumably, the pre-asymptotic to asymptotic regimes in rough fractures? What is the

118 asymptotic longitudinal scale for transport through rough fractures, if any? How robust is

119 CTRW-TPL for modeling the transport transition across scales? Is the relationship between $\beta$ in

120 the CTRW-TPL model and fracture roughness as established for the 2-D cases applicable for the

121 3-D cases? To this end, we first created synthetic fractures with aperture fields following typical

122 distributions as observed in natural fractures. Then we simulated solute transport through the

123 fractures using a computationally efficient quasi-3-D particle tracking random walk (PTRW)

124 algorithm which uses a 2-D velocity field computed through a modified Local Cubic Law. The

125 resultant 'experimental' breakthrough curves were then analyzed with the classical one-

126 dimensional (1-D) ADE and the CTRW-TPL. The statistical details of the heterogeneous flow

127 fields were examined in order to interpret the transport observations.

\section{2. Theoretical Background}

\section{$129 \quad$ 2.1. Advection dispersion equation}

The solute transport in shear flow through fractures can be upscaled to a 1-D advective-

131 dispersive process by using a bulk velocity and an effective dispersion coefficient, which reads 
as:

$$
\frac{\partial C}{\partial t}=-U_{\mathrm{ADE}} \nabla C+D_{\mathrm{ADE}} \nabla^{2} C
$$

133 where $C$ is upscaled solute concentration, $t$ is time, $U_{\mathrm{ADE}}$ is constant mean velocity, and $D_{\mathrm{ADE}}$ is 134 constant hydrodynamic dispersion coefficient. The inherent assumption behind the ADE is the 135 applicability of the Taylor (Fickian) dispersion theory (Taylor, 1953). That is, the travel time of 136 the solute is long enough so that the transverse gradients in solute concentration become 137 negligible; this threshold time is often called the "asymptotic time" (Koch and Brady, 1987). For 138 an idealized fracture with perfectly smooth surfaces (i.e., parallel plates), the asymptotic time 139 can be calculated from the aperture (Wang et al., 2012). However, fracture roughness or 140 heterogeneity may significantly lengthen the asymptotic time because it takes longer for the

141 transverse mixing to smooth out the longitudinal concentration gradients (Wang and Cardenas, 142 2014).

\subsection{Continuous time random walk theory}

The CTRW is fundamentally derived from the Master equation of statistical mechanics with appropriate memory functions to represent non-Fickian behavior (Berkowitz et al., 2006;

146 Cortis and Berkowitz, 2004). In 1-D Laplace space, the CTRW model reads as:

$$
p \tilde{C}(x, p)-C_{0}(x)=-\tilde{M}(p)\left[U_{\mathrm{CTRW}} \frac{\partial}{\partial x} \tilde{C}(x, p)-D_{\mathrm{CTRW}} \frac{\partial^{2}}{\partial x^{2}} \tilde{C}(x, p)\right]
$$

147 where the tilde represents a variable or function in the Laplace space, $p$ is the Laplace variable, $148 U_{\text {CTRW }}$ and $D_{\text {CTRW }}$ are transport velocity and dispersion coefficient in the context of the CTRW, 149 respectively, which may vary temporally according to the variations of local velocity fields caused by local heterogeneity (Berkowitz et al., 2006). Note that the $U_{\text {CTRW }}$ and $D_{\text {CTRW }}$ are not 
151 equivalent to $U_{\mathrm{ADE}}$ and $D_{\mathrm{ADE}}$ except under special cases. $\widetilde{M}(p)=\bar{t} p \frac{\widetilde{\psi}(p)}{1-\widetilde{\psi}(p)}$ represents the 152 memory function where $\tilde{\psi}(p)$ is the transition rate probability. The memory function connects 153 the micro-scale physics, e.g., the variation of the velocity field, to macro-scale non-Fickian 154 transport phenomena. This is because $\tilde{\psi}(p)$ represents micro-scale particle movements 155 dependent on the probability density function of the velocity field; it can also be viewed as the 156 origin for the non-Fickian transport. The CTRW framework has been extensively applied to various environments including, 158 for example, to characterize non-Fickian transport through porous media (Cortis and Berkowitz, 159 2004; Dentz et al., 2004), single fractures (Bauget and Fourar, 2008; Wang and Cardenas, 2014), 160 and fracture networks (Berkowitz and Scher, 1998). The cornerstone of CTRW is the 161 determination of $\tilde{\psi}(p)$. Cortis and Berkowitz (2004) considered three models for $\tilde{\psi}(p)$ : the 162 asymptotic model, the truncated power law model (TPL), and the modified exponential model. 163 Since the TPL reduces to the asymptotic model when the cut-off time is small, and since the 164 modified exponential model is not suitable for this study because it primarily describes a 165 random molecular diffusion system, the TPL was exclusively used here to model non-Fickian 166 solute transport.

The TPL model is described by:

$$
\tilde{\psi}(p)=\left(1+\tau_{2} p t_{1}\right)^{\beta} \exp \left(t_{1} p\right) \frac{\Gamma\left(-\beta, \tau_{2}^{-1}+t_{1} p\right)}{\Gamma\left(-\beta, \tau_{2}^{-1}\right)}
$$

168 where $t_{1}$ is the time for the onset of the power-law, $t_{2}$ is cut-off or truncation time 169 corresponding to when large-scale Fickian behavior begins or dominates, $\tau_{2}=t_{2} / t_{1}, \Gamma()$ is the 
170 incomplete Gamma function, and $\beta$ represents the degree of deviation from Fickian behavior

171 (Berkowitz et al., 2006; Cortis and Berkowitz, 2005; Dentz et al., 2004). The transport regimes

172 can be divided into (Berkowitz et al., 2006): (1) strongly non-Fickian transport where $0<\beta<1,(2)$

173 moderate non-Fickian transport where $1<\beta<2$, and (3) Fickian transport where $\beta>=2$ and the

174 CTRW-TPL converges to the ADE.

175

The CTRW-TPL potentially allows for capturing the transport process from non-Fickian

176 (pre-asymptotic) to Fickian (asymptotic) regimes while using consistent or scale-invariant

177 parameters, including the truncation time. However, recent studies highlight the non-

178 uniqueness of $\tilde{\psi}(p)$ during inverse modeling based on breakthrough curves (Hansen and

179 Berkowitz, 2014), thus requiring a careful manipulation of initial guesses of parameters and

180 assessment of the rationality of results calculated from the CTRW-TPL (Aubeneau et al., 2014;

181 Bauget and Fourar, 2008; Wang and Cardenas, 2014).

182 3. Methods

In this section, we first discuss the generation of the fractures and the calculation of

184 flow and transport fields within these fractures. This is followed by the methods we used for

185 analyzing the resulting fields.

$186 \quad 3.1$ Numerical simulations

$187 \quad 3.1 .1$ Two-dimensional flow modeling through synthetic fractures

Synthetic self-affine fractures with increasing lengths were generated through the

189 program SynFrac (Figure 1), which has been shown to reproduce the aperture distribution of

190 real fractures (Ogilvie et al., 2006). The synthetic fractures were prescribed with the same

191 arithmetic mean $\langle b\rangle$, standard deviation $\sigma_{b}(\sim 0.49\langle b\rangle)$, and correlation length $\lambda(=20<b>)$, 
192 where $b$ is aperture field. The smallest fracture size we considered was $50<b>\times 50<b>$ with $b$ 193 defined over a regular grid of $100 \times 100$ points over this fracture area. We increased the total 194 fracture length $L$ in increments of $100\langle b\rangle$ until the maximum of $1000\langle b\rangle$, i.e., the largest 195 fracture size is $1000<b>\times 50<b>$ with $2000 \times 100$ points (Figure 1 ). Note that with increasing fracture scales, the transverse mixing and spreading maybe 197 limited by the fracture lateral boundaries. The idealized case would be to maintain the domain 198 aspect ratio by increasing fracture width proportionally with increasing fracture length, but this 199 drastically increases the computational burden and requires resources that are beyond our 200 current computational capability. Moreover, simply increasing fracture length instead of width 201 is consistent with the CTRW theory and its previous classic validation studies using column 202 experiments (Cortis and Berkowitz, 2004). That is, the focus in this study agrees with the central 203 idea of CTRW of representing transitional transport behavior in the longitudinal direction. A predictive model for non-Fickian transport requires relating $\beta$ in the framework of 205 CTRW to fracture roughness; consequently, we generated synthetic fractures with aperture 206 field following both normal (Figure 2) and log-normal (Figure 3) distributions following random 207 fracture generation method (Brush and Thomson, 2003), because these types of distributions 208 are typically observed in natural fractures (Berkowitz, 2002). The correlation length $\lambda$ increases 209 from $10 \sim 100<b>$ with fixed $L(200<b>)$, and $\sigma_{b} /<b>$ varies with a broad range $0 \sim 2$ by 210 changing the magnitude of $\sigma_{b}$ or $\sigma_{\log (b)}$ while keeping $\langle b\rangle$ or $\langle\log (b)>$ constant. The small 211 fraction of contact areas $(b=0)$ when $\sigma_{b} /\langle b\rangle=2$ was artificially increased to be a constant $212(b=\langle b\rangle / 100)$. The variability of $\sigma_{b} /\langle b\rangle$ corresponds to that used in previous studies (Brush and 213 Thomson, 2003; Mourzenko et al., 1995). We then calculated the flow field through solving the 
214 modified Local Cubic Law and solute transport by implementing a particle tracking algorithm. For computational ease and efficiency, the Local Cubic Law (LCL), which is a simplified version of the Navier-Stokes equation, has been extensively applied to simulate vertically- or

217 aperture-integrated flow fields (Detwiler et al., 2002; Mourzenko et al., 1995). Though the LCL

218 offers computational advantages, it always overestimates volumetric flow rates (Brush and

219 Thomson, 2003; Wang et al., 2015; Zimmerman et al., 2004). To circumvent the shortcomings

220 of the $L C L$, here we used a recently developed modified $L C L(M L C L)$ to simulate the 2-D flow

221 fields (Wang et al., 2015), where flow was driven by a pressure gradient in the longitudinal

222 direction, and no-flux was applied for the other two transverse boundaries. The MLCL accounts

223 for fracture roughness, tortuosity, and weak inertial force and is described as:

$$
\nabla \cdot\left[\frac{T_{x}}{M} \frac{\partial p}{\partial x} \cos \left(\emptyset_{x}\right) \vec{\imath}+\frac{T_{y}}{M} \frac{\partial p}{\partial y} \cos \left(\emptyset_{y}\right) \vec{\jmath}\right]=0
$$

224 where $T$ is transmissivity, $p$ is pressure, $\phi$ is the flow orientation angle estimated from the 225 definition of local fracture tortuosity, and $M$ is a correction coefficient that accounts for local 226 fracture roughness and weak inertial force which have been previously calculated and 227 tabulated from results of equivalent-flux models of flow through wedges. Details of the MLCL 228 development and formulation are available in Wang et al. (2015) where it has also been shown 229 that solutions based on the MLCL are hydraulically equivalent to that from directly solving the 230 Navier-Stokes equations for 3-D fractures. The resultant 2-D MLCL-based velocity field served 231 as input for particle tracking simulations for solute transport.

232 3.1.2. Quasi-3-D solute transport modeling through particle tracking A quasi-3-D particle tracking random walk (PTRW) algorithm was used here to model 234 transport through the rough fractures, where the advection and diffusion of particles occur in 
235 the horizontal direction, while only diffusion occurs in the vertical (i.e., z) direction (James et al., 236 2005; Zheng et al., 2009). However, the exclusion of advection in the $z$ direction can be 237 complemented by including up/down movements according to the mean flow direction.

238 Moreover, the number of particles released at the inlet boundary is proportional to the local 239 flow rate, i.e., an idealized parabolic velocity profile. In the particle tracking method, the no-flux 240 condition is equivalent to an elastic reflection when a particle rebounds when it encounters the 241 lower, upper, and two lateral walls. In order to balance solution accuracy with a small time step 242 and computational efficiency with a large time step, we applied an adaptive time stepping 243 approach such that these two competing objectives can be achieved simultaneously as 244 explained in our previous study (Wang and Cardenas, 2015).

The details of our PTRW approach and its accuracy compared to direct numerical

246 simulations were described in Wang and Cardenas (2015); our previous study showed that the 247 PTRW using a vertically-integrated 2-D flow field solved through the MLCL accurately simulated 248 solute transport through 3-D rough and tortuous fractures (Wang and Cardenas, 2015), while 249 the PTRW with the conventional LCL may miss the overall transport behavior. Therefore, we 250 take advantage of our accurate and efficient approach and implemented the PTRW with MLCL 251 by instantaneously releasing about $N=1 \times 10^{4}$ particles at the inlet of increasingly larger 252 fractures. Simulation results were insensitive to the $N$ as we further increased $N$ (results were 253 not shown here). The modeling was implemented through MATLAB using parallel computing. 254 The forward transport modeling took 1 week 3 months to accomplish, where simulation time 255 depended on fracture scale, on a workstation with 16 processor cores and 80 Gb shared 256 memory. The PTRW made computation of BTCs possible even for the largest fracture; this 
257 would have been prohibitive with direct numerical simulation, i.e., solving the 3-D Navier-

258 Stokes equations and the advection-diffusion-equation. In our simulations, the Peclet number

$259\left(P_{e}=\langle u\rangle\langle b\rangle / D_{m}\right)$ ranges from 5.7 to 10.6 based on Table 1 , where $\langle u\rangle$ is mean flow velocity, $\langle b\rangle$

260 is arithmetic mean aperture, and $D_{m}$ is the diffusion coefficient. The transport corresponds to

261 the macrodispersion regime as defined by Detwiler et al. (2000). This study was designed to

262 eliminate strong Peclet number-dependence and thus exclusively identified the roughness'

263 effect on transport process across different length scales.

\section{3.2. Flow and solute transport analysis}

265 3.2.1. Statistical analysis of the flow field

267 exponential variogram model with nugget effect to the experimental variogram. The typical

268 exponential variogram $\gamma(h)$ model follows (Li et al., 2010):

$$
\gamma(h)=A\left[1-\exp \left(\frac{h}{\lambda}\right)\right]+\gamma_{0}
$$

269 where $\gamma_{0}$ is the nugget, $A$ is the sill, $h$ is the lag distance.

270 The spatial pattern of acceleration $\left(a_{t}\right)$ over time was estimated based on the ratio of

271 velocity difference to the travel time of individual particles:

$$
a_{t}=\frac{u_{n+1}-u_{n}}{t_{n+1}-t_{n}}
$$

272 where $n$ represents the current time step, and $n+1$ is future time step. The acceleration was

273 further used to analyze the probability density of its normalized standard deviation.

274 3.2.2. Calculation of breakthrough curves and residence time distributions 
To determine breakthrough curves, we continuously recorded the cumulative number

276 of particles $\left(N_{a}(t)\right)$ and arrival time at the outlet boundary. Dividing $N_{a}(t)$ by $N$ yielded the

277 cumulative fraction of particles (i.e., C) over time:

$$
C(t)=\frac{N_{a}(t)}{N}
$$

278

279

280

281

282

284

285

286

287

288

minimum rather than the global minimum. This potentially leads to parameter non-uniqueness

293 and identifiability issues with the CTRW-TPL. We therefore not only iteratively altered the initial

294 guesses based on the parameters from the ADE model to find the global minimum error, but

where $C(t)$ represents the 'real' or 'experimental' breakthrough curves (BTCs).

Residence time distributions (RTDs) provide an additional metric for examining non-

Fickian transport features: early arrival and heavy tailing. The RTD is calculated by taking the time derivative of the BTC.

\subsubsection{Inverse modeling of breakthrough curves}

Here, we employed the 1-D ADE and CTRW-TPL models to analyze the transport process transitioning from non-Fickian to Fickian regimes, and to establish a predictive model based on the physical properties of fractures. Two parameters $\left(U_{\mathrm{ADE}}\right.$ and $\left.D_{\mathrm{ADE}}\right)$ in the $\mathrm{ADE}$, and five parameters ( $U_{\mathrm{TPL}}, D_{\mathrm{TPL}}, t_{1}, t_{2}$, and $\left.\beta\right)$ in the CTRW-TPL were estimated through the 1-D inverse modeling module in the CTRW toolbox (Cortis and Berkowitz, 2005). Each BTC was analyzed independently. That is, no two or more BTCs were fit simultaneously.

The initial guess for unknowns in the ADE model were assumed to be constant, and the inversion was insensitive to the initial input. However, the initial guesses have a large impact in the inversion of the CTRW-TPL because the CTRW toolbox can easily arrive at a local error also maintained the same initial guesses across increasing scales to minimize this non- 
uniqueness issue. the curve fitting to the BTCs. The $E$ is:

$$
E_{i}=\sqrt{\sum_{n=1}^{N}\left(C_{1 D}^{i}-C_{n}\right)^{2}}
$$

299 300

where $N$ is the number of particles in the BTCs, $C_{1 D}^{i}$ represents the 1D BTCs fitted through the ADE and TPL models, where $i$ signifies either the ADE or the TPL, and $C$ is from the particle tracking. Errors from the ADE and TPL models are denoted as $E_{\mathrm{ADE}}$ and $E_{\mathrm{TPL}}$.

\section{Results and Discussions}

\subsection{Flow field spatial and statistical structure for fractures with increasing scales}

Non-Fickian transport fundamentally results from the spatial structure of the velocity field (Heidari and Li, 2014; Kang et al., 2014). And for fractures these are determined by the fracture roughness $\left(\sigma_{b} /\langle b\rangle\right)$ and the aperture correlation length $\lambda$. For fractures with increasing scales, we eliminated the effects of these factors by using a fixed $\sigma_{b} /\langle b\rangle$ and aperture $\lambda$. Thus, the velocity field structure should also remain similar. As expected, the velocity variograms showed a well-defined sill particularly for fractures with $L / \lambda>5$. The $\lambda$ of the velocity field consistently agreed with that prescribed for the aperture field $(\sim 20<b>$ ) (Figure 4a), indicating that the 2-D velocity fields were determined by the correlated aperture fields, a result one expects.

The statistical distributions (PDFs) of the particle velocities were also consistent for all fractures (Figure $4 b$ ). This is because $\left\langle b>\right.$ and $\sigma_{b}$, or roughness, was kept constant. The velocity PDFs exhibited a sharp and narrow peak about zero acceleration and two broad power-law tails; 
these features comprise a type of transition (intermittent) velocity structure (de Anna et al., 2013; Kang et al., 2014). The sharp peak about zero acceleration suggests that the particles would most likely encounter stagnant or low velocity zones, and long power-law tails indicate the likelihood of encountering velocity jumps from low to high values, or vice versa. Taken together, these illustrate the degree and nature of the flow and transport heterogeneity. Note that some transition velocity structures inherently control the temporal transition structure and thus generate non-Fickian transport behaviors (de Anna et al., 2013; Kang et al., 2014).

\subsection{Transport progression from Non-Fickian to Fickian regimes with increasing scales}

Early arrival and long tailing are two typical features of non-Fickian transport; these are clearly shown for fractures with $L / \lambda<30$ (Figures $5 a-5 d$ ). Perfect Fickian transport corresponds to an " $\mathrm{S}$ "-shaped curve centered about 1 pore volume (PV), where/when the normalized solute concentration $C^{\prime}=0.5$ is expected to be observed. This was not the case for most of the studied fractures. The experimental BTCs showed the progression of non-Fickian to Fickian transport as fracture scale longitudinally, i.e., the early arrivals and long tailing gradually disappeared at a $\log -\log$ scale (Figures $5 b$ and $5 d$ ). This is also demonstrated by the observation at a linear scale, since the expectation that $C^{\prime}=0.5$ at dimensionless time $=1 \mathrm{PV}$ was progressively satisfied and the ADE became more valid for fitting BTCs as fracture scale increased (Figures 5a-5b).

$$
\text { The RTDs further confirmed the transport progression from non-Fickian to Fickian with }
$$
increasing $L / \lambda$ (Figure 5e). The mode for the RTD was not only $<1$ PV for the relatively short fractures, but also increased with increasing $L / \lambda$, until this converged to 1 PV when the fracture was long enough which satisfied the Fickian transport condition. Moreover, bimodal RTDs were observed for the relatively short fractures, and these gradually evolved into inverted ' $U$ '-shaped 
curves. That is, the RTDs became more symmetric and tended to follow a Gaussian distribution with increasing fracture scales. Rigorously fitting certain portion of RTDs would yield different power law exponents (Figure 5e). The above-mentioned phenomena provided clear evidence that the early arrival and long tail disappear as fracture length increases.

The computational experiments clearly captured the transition from non-Fickian (preasymptotic) to Fickian (asymptotic) regimes in the presence of persistent intermittent velocity structure. These observations are in contrast to the expectation of persistent non-Fickian transport behavior with infinite Peclet number that needs longer time or length for transitioning into Fickian transport. For example, non-Fickian transport was persistently observed even after many hundreds of pore volumes for a porous medium in Kang et al. (2014). The different scaling effect on the earlier transport transition occurrence in this study is due to the inclusion of molecular diffusion in our PTRW algorithm (Berkowitz et al., 2006), which was ignored by Kang et al. (2014). We thus showed that the stationary intermittent velocity field within fractures can lead to Fickian transport once a threshold length scale is surpassed. The length scale for Fickian transport has been observed numerically through cubicpacked pores (Cardenas, 2009; Zhang and Lv, 2007), and is expected to be dependent on the magnitude of $P e$ and correlation length based on studies of porous media transport (Salles et al., 1993), but this has been rarely documented in rough fractures. Although the asymptotic time and length scales through fractures for the transport transition can be theoretically predicted through an idealized fracture (i.e., for the parallel plates model (Wang et al., 2012)), this prediction is complicated by fracture roughness which increases this length scale (Wang and Cardenas, 2014). Here, we determined based on visual inspection of the fits and the reduction 
of fitting errors (discussed further below) that rough-walled fractures should be at least of $\sim 35 \lambda$

361 long in order to transition to Fickian transport. This factor is greater than a previous

362 experimentally determined value of $20 \lambda$ by Detwiler et al. (2002); the difference may be largely

363 due to the different $P e$, which is fundamentally determined by the roughness of the fractures,

364 considered in previous studies compared to ours. Moreover, this factor is smaller than the

365 theoretical value (10Pe $\lambda$ ) estimated for porous media (Salles et al., 1993). Future research

366 efforts should systematically explore the combined roles of $P e$ and aperture $\lambda$ on controlling

367 the length scale for transport regime transition through fractures.

\subsection{Theoretical prediction and assessment of transport transition}

The ADE model as expected failed to capture typical non-Fickian transport features

370 (early arrival and long tails) for the relatively short fractures which led to large fitting errors. But

371 the ADE model was gradually able to match the experimental BTCs as $L / \lambda$ increased (Figures $5 a-$

$3725 b)$ with concurrent reductions in error (Table 1$)$. The fitted velocity $\left(U_{A D E}\right)$ agreed well with

373 mean flow velocity $(U)$, which is calculated as the ratio of $L$ to the PV (Table 1 ). The fitted $D_{\mathrm{ADE}}$

374 increased initially with increasing $L / \lambda$ but then decreased with further increases in $L / \lambda$ (Figure 6

375 and Table 1). Thus, the inverse modeling analysis with the ADE led to somewhat erratic

376 behavior in $D_{\mathrm{ADE}}$ and did not show a clear progression towards the asymptotic regime where

$377 D_{\mathrm{ADE}}$ should be stationary.

378 Compared to the ADE, the CTRW-TPL framework was able to fit the experimental BTCS

379 across scales and transport regimes from non-Fickian to Fickian (Figures 5c-5d, Table 1). The

380 errors remained small except for the shortest fracture with $L / \lambda=2.5$. For all other fracture

381 length cases, the CTRW-TPL showed similar if not smaller errors than the smallest error 
382 achieved with the $\mathrm{ADE}$ even in the asymptotic regime. At each scale $E_{\mathrm{TPL}}$ is always less than $E_{\mathrm{ADE}}$.

383 However, for the shortest fracture, errors were large for both cases. This is because the ADE

384 and the CTRW-TPL framework completely miss and in fact cannot handle the bimodal RTDs

385 (Figure 5e). Unlike the ADE which would require adaption to include an integro-differential

386 term to model the progression of non-Fickian to Fickian transport, the CTRW-TPL reproduced

387 the bulk transport behavior with a more or less consistent set of parameters that was

388 insensitive to the scales (Table 1), because the exponential function dominates over the power

389 law at large scales as shown in equation (3). That is, the power law plays a nominal role even

390 though it may be still applicable for certain portion of RTDs with varying power law exponents

391 (Figure 5e).

Non-uniqueness during the inversion process can be a problem with CTRW (Hansen and

393 Berkowitz, 2014), especially when transport converges to the Fickian regime. However, here we

394 observed the stabilization of $\beta$ and $t_{2}$ for fractures with increasing $L / \lambda$ (Figure 6 ) across Fickian

395 and non-Fickian transport regimes given that fracture roughness remains constant. In our cases,

396 the CTRW-TPL framework was sensitive to these two parameters and less so to $t_{1}$, since the

397 latter changed a little bit with increasing scales but remained small compared to $t_{2}$. $\beta$ fluctuated

398 slightly about $\sim 1.13$. Moreover, $t_{2}$ converged and stabilized quickly to around $10^{3.4} \mathrm{~s}$

399 (disregarding the fracture $L / \lambda=2.5$ ). Thus the $\beta$ and $t_{2}$ that resulted from the inverse modeling

400 are truly reflective of the local micro-scale processes and is insensitive to the domain size. This

401 implies that incorporation of transport process at one scale may improve the prediction of $\beta$ at

402 other scales if fracture roughness is stationary. Moreover it showed that $\beta$ may be insensitive to

403 the fracture correlation length as we previously showed for 2-D fractures (Wang and Cardenas, 
2014). Based on the magnitude of $\beta$, a moderate non-Fickian transport was observed; this

405

indicated that the transport velocity is constant and that the dispersion coefficient scales as a power law over time. Therefore, $U_{\text {TPL }}$ remains fairly constant but $D_{\text {TPL }}$ varies with increasing

$407 L / \lambda$ for our numerical results (Table 1 ).

\subsection{Impact of fracture roughness and aperture correlation length on effective solute}

transport velocity

Fluid velocity is known to be different from transport velocity in fractures (Silliman, 1989;

Tsang, 1992; Zheng et al., 2008). Depending on the anisotropy of the aperture field, transport

412 velocity could be either greater or smaller than fluid velocity if flow direction is parallel to or

413 perpendicular to the aperture variations, respectively (Silliman, 1989). For an isotropic case,

414 theoretical analysis indicates that transport velocity is retarded by fracture roughness $\left(\sigma_{b} /<b>\right)$

415 compared to fluid velocity, and the retardation coefficient could be solely predicted based on

$416 \sigma_{b} /\langle b\rangle$ (Silliman, 1989). Our results were consistent with this theory that transport velocity is

417 always less than fluid velocity as our synthetic fractures were isotropic (Figure 7). Unlike

418 previous studies, we found that the deviations between fluid and transport velocities are

419 dependent on both $\sigma_{b} /\langle b\rangle$ and $\lambda$. For short-correlation fractures, the ratio of fluid velocity to

420 transport velocity increases exponentially with $\sigma_{b} /\langle b\rangle$. However, this incremental rate of

421 velocity ratio with respect to $\sigma_{b} /\langle b\rangle$ decreases as $\lambda / L$ increases, and eventually become

422 insensitive to $\sigma_{b} /\langle b\rangle$ especially when $\left.\lambda / L\right\rangle=0.8$ (Figure 7). This is most likely due to the fact

423 that the aperture field with a large $\lambda / L$ tends to create preferential paths, and these fast flow

424 paths are spatially invariant across a broad range of $\sigma_{b} /\langle b\rangle$. Future research could explore a

425 quantitative relationship between velocity ratio and $\sigma_{b} /\langle b\rangle$ and $\lambda / L$, such that the effective 
426 transport velocity can be empirically predicted based on fluid velocity.

427

428

429

430

431

432

433

434

435

436

437

438

439

440

441

442

443

444

445

446

447

\subsection{Dependence of non-Fickian magnitude on 3-D fracture heterogeneity}

Non-Fickian transport can be attributed to incomplete local mixing or to mixing failing to reach time and length scales that satisfy Fickian dispersion theory (Dentz et al., 2011; Le Borgne et al., 2011). Rough fracture walls (i.e., $\sigma_{b} /\langle b\rangle$ ) create immobile zones which induce incomplete local mixing and lengthen the residence time of solutes. Moreover, $\lambda$ affects the length scales that satisfy Fickian dispersion as discussed in section 4.3. In any case, non-Fickian transport through 2-D fractures can be accurately captured by the CTRW-TPL model (Wang and Cardenas, 2014), where the degree of deviation of non-Fickian from Fickian transport can be represented by $\beta$.

Our previous study on non-Fickian transport through 2-D fractures showed that $\beta$ is proportional to $\sigma_{b} /\langle b\rangle$, but it is insensitive to $\lambda / L$ (Wang and Cardenas, 2014). Yet, the prediction of non-Fickian transport through 3-D fractures remains elusive particularly with respect to $\sigma_{b} /\langle b\rangle$ and $\lambda$. To this end, we inversely estimated $\beta$ based on the "experimental" breakthrough curves through 3-D rough and tortuous fractures, where aperture fields follow either normal (Figure 2) or log-normal distributions (Figure 3). Similar to transport behavior through 2-D fractures, for 3-D cases, $\beta$ is found to be linearly proportional to $\sigma_{b} /\langle b\rangle$ despite of variability in $\lambda / L$ (Figure 8). Note that the slope and intercept of the regressed linear equation are different from that for 2-D cases. This might be due to additional transverse dispersion occurring in 3-D rough fractures. Specifically, $\beta$ in a 3-D case was observed to be smaller than that in a 2-D case for given $\sigma_{b} \mid\langle b\rangle$, and resulted in non-Fickian transport with relatively heavier tailing. Moreover, $\beta$ decreased rapidly when $\sigma_{b} /\langle b\rangle<0.1(\beta=2$ for parallel plates since Fickian 
448 transport occurs within a relatively short length) which was not observed in a 2-D case (Figure

449 8). This shows that a small change in $\sigma_{b} /\langle b\rangle$ may significantly change the transverse dispersion

450 process which in turn contributes to longer solute residence time through 3-D fractures.

As expected, $\beta$ is more sensitive to $\sigma_{b} /\langle b\rangle$ rather than $\lambda$ for both 2-D (Wang and

452 Cardenas, 2014) and 3-D (Figure 8) fractures. This is because $\beta$ is naturally a scale-insensitive

453 parameter, as discussed in the section 4.3. As long as $L / \lambda$ is greater than 2.5 , which is when $\beta$

454 becomes stable (Figure 6), the variation of $\lambda$ will not significantly affect $\beta$ (Figure 8 ). Our study

455 further showed the robustness and applicability of CTRW-TPL model for predicting non-Fickian

456 transport by directly linking it to measurable physical properties of fractures across different

457 scales.

458

459

460

461

462

463

464

465

466

467

468

469

\subsection{Limitations of current study}

Although the ability of the CTRW for predicting the transport transition from non-Fickian to Fickian with increasing scales has been validated here with measurable properties of single fractures, the scaling effects on transport behavior in a fractured system could be further complicated by fracture density and orientations; these present an additional challenge in characterizing non-Fickian transport at increasing scales (Berkowitz, 2002; Brown, 1987;

Neuman, 2005). Moreover, our analysis takes into account only the effect of the longitudinal scale of transport. Ideally, the fractures can be made wider in the transverse direction as the longitudinal $L$ is increased. Thus, our analysis assumes that the transverse length is sufficiently large such that it does not add additional pre-asymptotic mixing. Given that $\beta$ apparently stabilizes at $L / \lambda>2.5$, this assumption is reasonable since asymptotic mixing in the transverse direction for a solute released uniformly is typically achieved even sooner. 
471 investigated here. Increasing inertial force not only leads to the failure of Darcy' law in

472 describing fluid flow (Zhou et al., 2016; Zimmerman et al., 2004), but also results in the failure

473 of the ADE in describing transport, which can be attributed to the growth of the immobile zones

474 as shown by 2-D studies (Bolster et al., 2014; Cardenas et al., 2009). Unlike 2-D cases, recent

475 experimental results further showed a shortened tailing with increasing Reynolds number $(R e)$

476 within laterally extensive 3-D rough fractures (Lee et al., 2015). However, transport across

477 increasing $R e$ in natural 3-D rough fractures is still poorly understood. Therefore, appropriate

478 prediction of non-Fickian behavior across a broad range of $R e$ remains an open challenge. The

479 aspects mentioned above are beyond the scope of the current study.

$480 \quad$ 5. Summary and Conclusions

481

Flow and transport through fractures is complex with seemingly ubiquitous non-Fickian

482 transport. In this study, we first illustrated the progression of transport from pre-asymptotic

483 (non-Fickian) to asymptotic (Fickian) transport through 3-D rough-walled synthetic fractures by

484 modeling experiments conducted at incrementally increasing scales but where fracture

485 roughness and aperture correlation length remained constant. The solute transport was

486 simulated through a quasi-3-D particle tracking random walk algorithm with a 2-D flow field

487 calculated through a modified local cubic law. This resulted in a series of experimental

488 breakthrough curves corresponding to each fracture scale. The solute breakthrough curves and

489 residence time distributions clearly showed the transition from non-Fickian to Fickian transport

490 in the presence of an intermittent velocity structure; the transport transition is expected to

491 occur later when molecular diffusion is ignored. The Fickian advection-dispersion equation 
492

493

494

495

496

497

498

499

500

501 502 scales.

503

504

505

506

507

508

509

510

511

512

513

.

expectedly failed to capture transport for the relatively short fractures and resulted in erratic estimates for the dispersion coefficient. Analysis of the breakthrough curves with the continuous time random walk (CTRW) with truncated power law resulted in a set of transport parameters that were insensitive to fracture scale, even for the shorter fractures and through the transport regime transition. Moreover, we implemented additional particle tracking experiments through fractures with varying roughness and correlation length, where aperture fields follow either normal or log-normal distributions. We found that the parameter $\beta$ in the CTRW model is linearly dependent on fracture roughness but is insensitive to aperture correlation length. Therefore, the CTRW model parameters determined from measurable physical properties of fractures at various scales can be used for transport prediction at other

Acknowledgements. This material is based upon work supported as part of the Center for Frontiers of Subsurface Energy Security (CFSES) at the University of Texas at Austin, an Energy Frontier Research Center funded by the U.S. Department of Energy, Office of Science, Office of Basic Energy Sciences under Award Number DE-SC0001114. Additional support was provided by the Geology Foundation of the University of Texas. We note that there is no data sharing issue because all results retrieved from numerical simulations are provided in the figures and table. Fracture geometries and breakthrough curve data are freely available from the authors upon request. We thank Editor Christian Griebler and two anonymous reviewers for their insightful comments in evaluating this manuscript. 


\section{References}

515 Aubeneau, A.F., Hanrahan, B., Bolster, D. and Tank, J.L., 2014. Substrate size and heterogeneity control anomalous transport in small streams. Geophys. Res. Lett., 41(23): 8335-8341.

517 Bauget, F. and Fourar, M., 2008. Non-Fickian dispersion in a single fracture. J. Contam. Hydrol., 100(3-4): 137-148.

Berkowitz, B., 2002. Characterizing flow and transport in fractured geological media: A review. Adv. Water Resour., 25(8-12): 861-884.

521

522

523

524

525

526

527

528

529

530

531

532

533

534

535
Berkowitz, B., Cortis, A., Dentz, M. and Scher, H., 2006. Modeling non-Fickian transport in geological formations as a continuous time random walk. Rev. Geophys., 44(2): RG2003.

Berkowitz, B. and Scher, H., 1998. Theory of anomalous chemical transport in random fracture networks. Phys. Rev. E, 57(5): 5858-5869.

Berkowitz, B. and Scher, H., 2010. Anomalous transport in correlated velocity fields. Phys. Rev. E, 81(1): 011128.

Bolster, D., Méheust, Y., Le Borgne, T., Bouquain, J. and Davy, P., 2014. Modeling preasymptotic transport in flows with significant inertial and trapping effects-The importance of velocity correlations and a spatial markov model. Adv. Water Resour., 70: 89-103.

Bouquain, J., Meheust, Y., Bolster, D. and Davy, P., 2012. The impact of inertial effects on solute dispersion in a channel with periodically varying aperture. Phys. Fluids, 24(8): 083602-17.

Brown, S.R., 1987. Fluid flow through rock joints: The effect of surface roughness. J. Geophys. Res., 92(B2): 1337-1347.

Brush, D.J. and Thomson, N.R., 2003. Fluid flow in synthetic rough-walled fractures: NavierStokes, Stokes, and local cubic law simulations. Water Resour. Res., 39(4): 1085. 
536 Candela, T. et al., 2012. Roughness of fault surfaces over nine decades of length scales. J.

537

538

539

540

541

542

543

544

545

546

547

548

549

550

551

552

553

554

555

Geophys. Res., 117(B8): B08409.

Cardenas, M.B., 2009. Direct simulation of pore level Fickian dispersion scale for transport through dense cubic packed spheres with vortices. Geochem. Geophys. Geosyst., 10(12): Q12014.

Cardenas, M.B., Slottke, D.T., Ketcham, R.A. and Sharp, J.M., Jr., 2007. Navier-Stokes flow and transport simulations using real fractures shows heavy tailing due to eddies. Geophys. Res. Lett., 34(14): L14404.

Cardenas, M.B., Slottke, D.T., Ketcham, R.A. and Sharp, J.M., Jr., 2009. Effects of inertia and directionality on flow and transport in a rough asymmetric fracture. J. Geophys. Res., 114(B6): B06204.

Cortis, A. and Berkowitz, B., 2004. Anomalous transport in classical soil and sand columns. Soil Sci. Soc. Am. J., 68(5): 1539-1548.

Cortis, A. and Berkowitz, B., 2005. Computing "anomalous" contaminant transport in porous media: The CTRW MATLAB Toolbox. Ground Water, 43(6): 947-950.

Cortis, A., Gallo, C., Scher, H. and Berkowitz, B., 2004. Numerical simulation of non-Fickian transport in geological formations with multiple-scale heterogeneities. Water Resour. Res., 40(4): W04209.

de Anna, P. et al., 2013. Flow intermittency, dispersion, and correlated continuous time random walks in porous media. Phys. Rev. Lett., 110(18): 184502. 
Dentz, M., Cortis, A., Scher, H. and Berkowitz, B., 2004. Time behavior of solute transport in heterogeneous media: transition from anomalous to normal transport. Adv. Water Resour., 27(2): 155-173.

Dentz, M., Le Borgne, T., Englert, A. and Bijeljic, B., 2011. Mixing, spreading and reaction in heterogeneous media: A brief review. J. Contam. Hydrol., 120-121(0): 1-17.

Detwiler, R.L., Rajaram, H. and Glass, R.J., 2000. Solute transport in variable-aperture fractures: An investigation of the relative importance of Taylor dispersion and macrodispersion. Water Resour. Res., 36(7): 1611-1625.

Detwiler, R.L., Rajaram, H. and Glass, R.J., 2002. Experimental and simulated solute transport in a partially-saturated, variable-aperture fracture. Geophys. Res. Lett., 29(8): 113-1-113-4.

Hansen, S.K. and Berkowitz, B., 2014. Interpretation and nonuniqueness of CTRW transition distributions: Insights from an alternative solute transport formulation. Adv. Water Resour., 74(0): 54-63.

Heidari, P. and Li, L., 2014. Solute transport in low-heterogeneity sandboxes: The role of correlation length and permeability variance. Water Resour. Res., 50(10): 8240-8264.

James, S.C., Bilezikjian, T. and Chrysikopoulos, C., 2005. Contaminant transport in a fracture with spatially variable aperture in the presence of monodisperse and polydisperse colloids. Stoch. Environ. Res. Ris. Assess., 19(4): 266-279.

James, S.C. and Chrysikopoulos, C.V., 2000. Transport of polydisperse colloids in a saturated fracture with spatially variable aperture. Water Resour. Res., 36(6): 1457-1465.

Kang, P.K. et al., 2014. Pore-scale intermittent velocity structure underpinning anomalous transport through 3-D porous media. Geophys. Res. Lett., 41(17): 6184-6190. 
578 Kang, P.K., Dentz, M., Le Borgne, T. and Juanes, R., 2011. Spatial markov model of anomalous transport through random lattice networks. Phys. Rev. Lett., 107(18): 180602.

Keller, A.A., Roberts, P.V. and Blunt, M.J., 1999. Effect of fracture aperture variations on the dispersion of contaminants. Water Resour. Res., 35(1): 55-63.

Koch, D.L. and Brady, J.F., 1987. A non-local description of advection-diffusion with application to dispersion in porous media. J. Fluid. Mech., 180: 387-403.

Le Borgne, T., Dentz, M. and Carrera, J., 2008. Lagrangian statistical model for transport in highly heterogeneous velocity fields. Phys. Rev. Lett., 101(9): 090601.

Le Borgne, T. et al., 2011. Persistence of incomplete mixing: A key to anomalous transport. Phys. Rev. E, 84(1): 015301.

Lee, S.H., Yeo, I.W., Lee, K.K. and Detwiler, R.L., 2015. Tail shortening with developing eddies in a rough-walled rock fracture. Geophys. Res. Lett., 42(15): 6340-6347.

Li, L., Zhou, H. and Jaime Gómez-Hernández, J., 2010. Steady-state saturated groundwater flow modeling with full tensor conductivities using finite differences. Comput. Geosci., 36(10): 1211-1223.

Majdalani, S., Chazarin, J.P., Delenne, C. and Guinot, V., 2015. Solute transport in periodical heterogeneous porous media: Importance of observation scale and experimental sampling. J. Hydrol., 520(0): 52-60.

McInnis, D.P., Bolster, D. and Maurice, P.A., 2014. Natural organic matter transport modeling with a continuous time random walk approach. Environ. Eng. Sci., 31(2): 98-106.

Mourzenko, V.V., Thovert, J.F. and Adler, P.M., 1995. Permeability of a single fracture - Validity of the Reynolds-Equation. Journal De Physique li, 5(3): 465-482. 
600

601

602

603

604

605

606

607

608

609

610

611

612

613

614

615

616

617

618

619

620

621

Mukhopadhyay, S., Liu, H.H., Spycher, N. and Kennedy, B.M., 2013. Impact of fluid-rock chemical interactions on tracer transport in fractured rocks. J. Contam. Hydrol., 154(0): $42-52$

Neuman, S.P., 2005. Trends, prospects and challenges in quantifying flow and transport through fractured rocks. Hydrogeol. J., 13(1): 124-147.

Neuman, S.P. and Tartakovsky, D.M., 2009. Perspective on theories of non-Fickian transport in heterogeneous media. Adv. Water Resour., 32(5): 670-680.

Nowamooz, A., Radilla, G., Fourar, M. and Berkowitz, B., 2013. Non-Fickian transport in transparent replicas of rough-walled rock fractures. Transport Porous Med., 98(3): 651682.

Ogilvie, S.R., Isakov, E. and Glover, P.W.J., 2006. Fluid flow through rough fractures in rocks. II: A new matching model for rough rock fractures. Earth Planet. Sc. Lett., 241(3-4): 454-465.

Roubinet, D., de Dreuzy, J.R. and Tartakovsky, D.M., 2012. Semi-analytical solutions for solute transport and exchange in fractured porous media. Water Resour. Res., 48(1): W01542.

Salles, J. et al., 1993. Taylor dispersion in porous media. Determination of the dispersion tensor. Phys. Fluids A: Fluid, 5(10): 2348-2376.

Silliman, S.E., 1989. An interpretation of the difference between aperture estimates derived from hydraulic and tracer tests in a single fracture. Water Resour. Res., 25(10): 22752283.

Sund, N.L., Bolster, D. and Dawson, C., 2015. Upscaling transport of a reacting solute through a peridocially converging-diverging channel at pre-asymptotic times. J. Contam. Hydrol., 182: 1-15. 
622 Taylor, G., 1953. Dispersion of soluble matter in solvent flowing slowly through a tube. P. Roy.

623

624

625

626

627

628

629

630

631

632

633

634

635

636

637

638

639

640

641

642

643

Soc. Lond. A. Mat., 219(1137): 186-203.

Tsang, Y.W., 1992. Usage of "equivalent apertures" for rock fractures as derived from hydraulic and tracer tests. Water Resour. Res., 28(5): 1451-1455.

Wang, L. and Cardenas, M.B., 2014. Non-Fickian transport through two-dimensional rough fractures: Assessment and prediction. Water Resour. Res., 50(2): 871-884.

Wang, L. and Cardenas, M.B., 2015. An efficient quasi-3D particle tracking-based approach for transport through fractures with application to dynamic dispersion calculation. J. Contam. Hydrol., 179(0): 47-54.

Wang, L., Cardenas, M.B., Deng, W. and Bennett, P.C., 2012. Theory for dynamic longitudinal dispersion in fractures and rivers with Poiseuille flow. Geophys. Res. Lett., 39(5): L05401.

Wang, L.C., Cardenas, M.B., Slottke, D.T., Ketcham, R.A. and Sharp, J.M., 2015. Modification of the Local Cubic Law of fracture flow for weak inertia, tortuosity, and roughness. Water Resour. Res., 51(4): 2064-2080.

Zhang, X. and Lv, M., 2007. Persistence of anomalous dispersion in uniform porous media demonstrated by pore-scale simulations. Water Resour. Res., 43(7): W07437.

Zheng, Q., Dickson, S. and Guo, Y., 2009. Influence of aperture field heterogeneity and anisotropy on dispersion regimes and dispersivity in single fractures. J. Geophys. Res., 114(B3): B03205.

Zheng, Q., Dickson, S.E. and Guo, Y., 2008. On the appropriate "equivalent aperture" for the description of solute transport in single fractures: Laboratory-scale experiments. Water Resour. Res., 44(4): W04502. 
644 Zhou, J.-Q., Hu, S.-H., Chen, Y.-F., Wang, M. and Zhou, C.-B., 2016. The friction factor in the 645 Forchheimer Equation for rock fractures. Rock Mech. Rock Eng.: 1-14.

646

647

648

649

650

651

652

653

654

655

656 where $L$ is total length of fractures as shown in Figure 1, PV denotes pore volume.

\begin{tabular}{ccccccccccc}
$\begin{array}{c}\text { Relative } \\
\text { length } \\
L / \lambda\end{array}$ & $\begin{array}{c}U_{\mathrm{ADE}} \\
\times 10^{-5}\end{array}$ & $\begin{array}{c}D_{\mathrm{ADE}} \\
\times 10^{-7}\end{array}$ & $\begin{array}{c}E_{\mathrm{ADE}} \\
{[\mathrm{m} / \mathrm{s}]}\end{array}$ & $\begin{array}{c}U=L / \mathrm{PV} \\
\times 10^{-5}\end{array}$ & $\begin{array}{c}U_{\mathrm{TPL}} \\
\times 10^{-4}\end{array}$ & $\begin{array}{c}D_{\mathrm{TPL}} \\
\times 10^{-7}\end{array}$ & $\beta$ & $t_{1}$ & $t_{2}$ & $E_{\mathrm{TPL}}$ \\
{$\left[\mathrm{m}^{2}\right]$} & {$[-]$} & {$[\mathrm{m} / \mathrm{s}]$} & {$[\mathrm{m} / \mathrm{s}]$} & $\begin{array}{c}\mathrm{m}^{2} / \mathrm{s} \\
]\end{array}$ & {$[-]$} & {$[\mathrm{s}]$} & {$[\mathrm{s}]$} & {$[-]$} \\
\hline 2.5 & 2.28 & 1.10 & 3.37 & 3.19 & 0.38 & 1.80 & 1.30 & $10^{-1.63}$ & $10^{-0.11}$ & 3.37 \\
5.0 & 4.23 & 1.65 & 1.56 & 4.41 & 2.91 & 0.22 & 1.00 & $10^{-0.20}$ & $10^{3.33}$ & 0.54 \\
10 & 4.25 & 1.44 & 2.05 & 4.23 & 1.87 & 0.29 & 1.15 & $10^{-0.21}$ & $10^{3.61}$ & 0.25 \\
15 & 3.39 & 1.98 & 0.75 & 3.37 & 1.49 & 3.31 & 1.14 & $10^{-0.06}$ & $10^{3.49}$ & 0.47 \\
20 & 3.93 & 2.58 & 0.71 & 3.97 & 1.74 & 6.39 & 1.17 & $10^{-0.73}$ & $10^{3.53}$ & 0.73 \\
25 & 3.99 & 2.57 & 2.48 & 3.76 & 1.99 & 0.71 & 1.14 & $10^{-0.36}$ & $10^{3.89}$ & 0.36 \\
30 & 3.79 & 1.55 & 1.51 & 3.65 & 1.62 & 0.70 & 1.17 & $10^{-0.44}$ & $10^{3.68}$ & 0.46 \\
35 & 3.95 & 1.30 & 0.91 & 4.09 & 1.76 & 0.89 & 1.14 & $10^{-0.46}$ & $10^{3.25}$ & 0.22 \\
50 & 3.03 & 1.04 & 0.86 & 3.08 & 1.52 & 1.09 & 1.12 & $10^{-0.69}$ & $10^{3.36}$ & 0.19
\end{tabular}


Figure captions

659 Figure 1. Normally distributed synthetic aperture fields used in this study. The fractures have 660 the same arithmetic mean $\langle b\rangle$, standard deviation of aperture field $b$, and correlation length $\lambda$

661 across varying fracture total length $L$. The fractures were generated through the SynFrac

662 program (Ogilvie et al., 2006).

663

664

Figure 2. Examples of fractures with aperture field following normal distribution with different

665 roughness $\left(\sigma_{b} /\langle b\rangle\right)$ and correlation length $(\lambda)$, where $b$ is apparent aperture with standard

666 deviation $\sigma_{b}$ of aperture and arithmetic mean aperture $\langle b\rangle, L$ is the total length of fractures.

667

668 Figure 3. Examples of fractures with aperture field following log-normal distribution with 669 different roughness $\left(\sigma_{b} /\langle b\rangle\right)$ and correlation length $(\lambda)$, where $b$ is apparent aperture with 670 standard deviation $\sigma_{b}$ of aperture and arithmetic mean aperture $\langle b\rangle, L$ is the total length of 671 fractures.

672

673 Figure 4. Statistical properties of the flow field for fractures with different length. (a)

674 Experimental variogram $\gamma(h)$ for the longitudinal velocity field for the studied fractures; $h$ is lag

675 distance and $\langle b\rangle$ is the arithmetic mean of aperture field $b$. (b) Probability distribution function 676 (PDF) of normalized Lagrangian acceleration of particles in the $x$ direction $\left(a_{t}(x)\right)$, where $a_{t}$ is 677 acceleration with its standard deviation $\left(\sigma_{a t}\right), L$ is total length, and $\lambda$ is the correlation length of 678 aperture field.

679 
680 Figure 5. Experimental breakthrough curves (BTCs) generated from particle tracking (hollow

681 circles), and inverse or fitted BTCs from solving the advection-dispersion equation (a-b, solid 682 lines) and continuous random walk (c-d, solid lines) for fractures with increasing scales (total

683 length $L /$ correlation length $\lambda$ ). Each BTC group was shifted by 1 pore volume $\tau$ with each

684 incremental increase in scale for visual purposes in a) and c). The corresponding residence time 685 distributions (RTDs) are shown in (e) with the fitted power law shown as black lines. $C^{\prime}$ 686 represents normalized concentration.

687

688 Figure 6 . The scale-dependence of transport parameters: 1-D ADE dispersion coefficient $\left(D_{\mathrm{ADE}}\right)$, 689 CTRW-TPL $\beta$, and CTRW-TPL truncation time $t_{2}$.

690

691 Figure 7 . The ratio of fluid velocity $\left(\mathrm{V}_{\text {Fluid }}\right)$ to effective transport velocity $\left(\mathrm{V}_{\text {Transport }}\right)$ is a function 692 of fracture roughness $\left(\sigma_{b} /\langle b\rangle\right)$ and aperture correlation length $(\lambda)$, where $V_{\text {Transport }}$ is obtained 693 by fitting breakthrough curves to the advection-dispersion equation, $b$ is apparent aperture 694 with standard deviation $\sigma_{b}$ of aperture and arithmetic mean aperture $\langle b\rangle, L$ is the total length 695 of fracture.

697 Figure 8. Dependence of parameter $\beta$ in the CTRW-TPL model on fracture roughness $\left(\sigma_{b} /\langle b\rangle\right)$, 698 where $b$ is apparent aperture with standard deviation $\sigma_{b}$ of aperture and arithmetic mean 699 aperture $\langle b\rangle, L$ is the total length of studied fracture. Color represents different aperture 700 correlation length $(\lambda)$ following normal and log-normal distributions as shown by hollow circle 
701 and asterisk, respectively, filled blue circle refers to the ideal parallel plates model. The black 702 line is the linear fit for $\beta$ with $\sigma_{b} /\langle b\rangle$. 


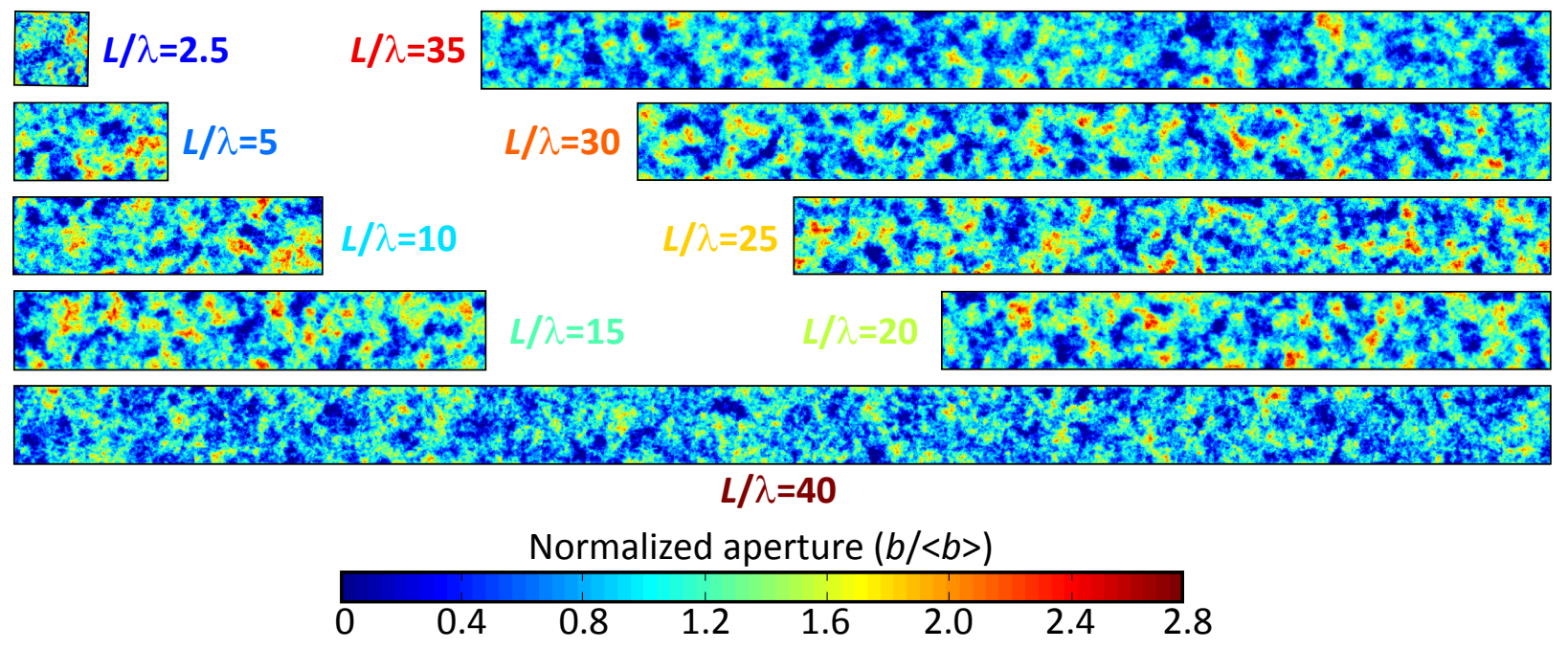

Figure 1. Normally distributed synthetic aperture fields used in this study. The fractures have the same arithmetic mean $\langle b\rangle$, standard deviation of aperture $b$, and correlation length $\lambda$ across varying fracture total length $L$. The fractures were generated through the SynFrac program [Ogilvie et al., 2006]. 


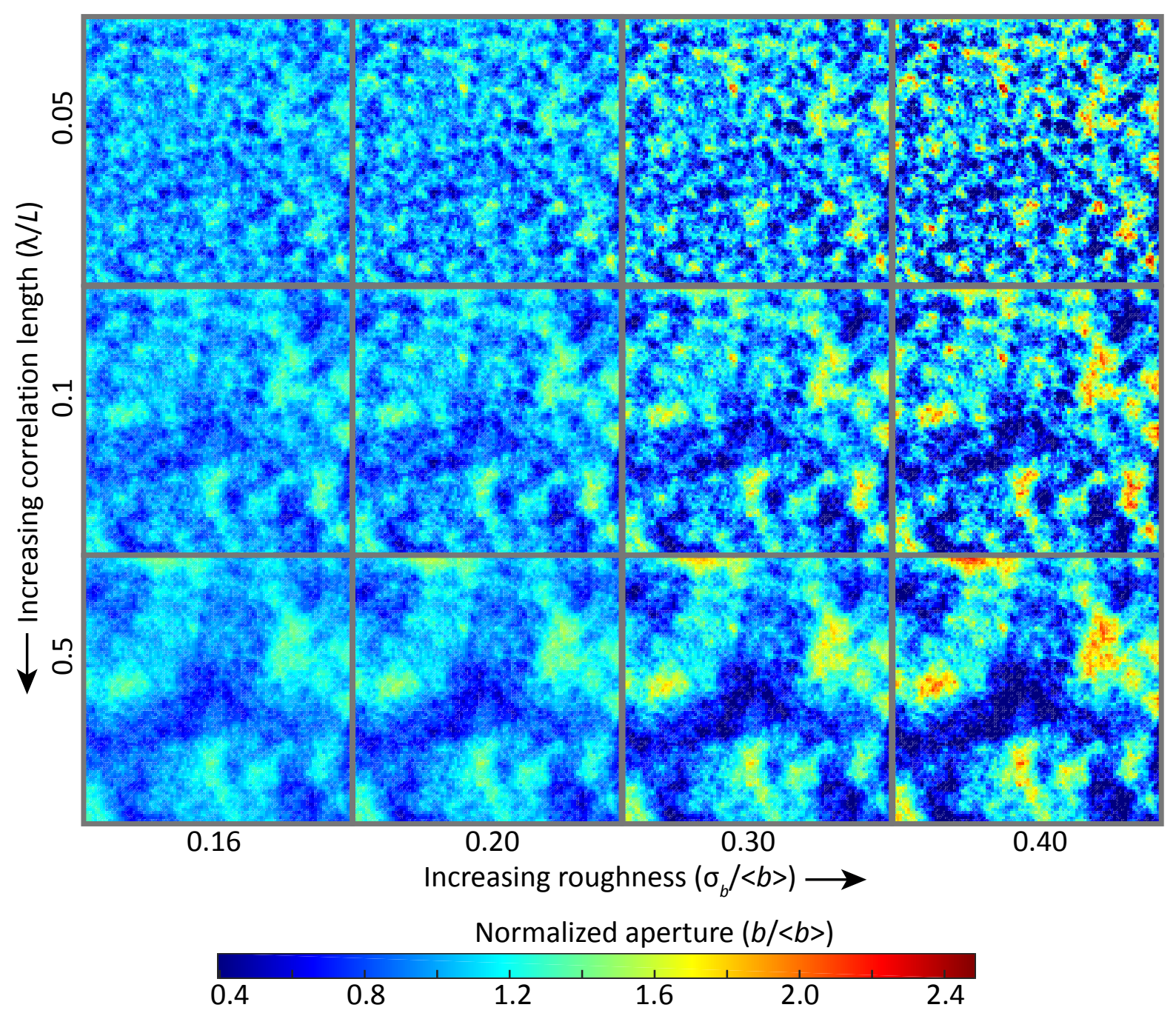

Figure 2. Examples of fractures with aperture field following normal distribution with different roughness $\left(\sigma_{b} /\langle b\rangle\right)$ and correlation length $(\lambda)$, where $b$ is apparent aperture with standard deviation $\sigma_{b}$ of aperture and arithmetic mean aperture $\langle b\rangle, L$ is the total length of fractures. 


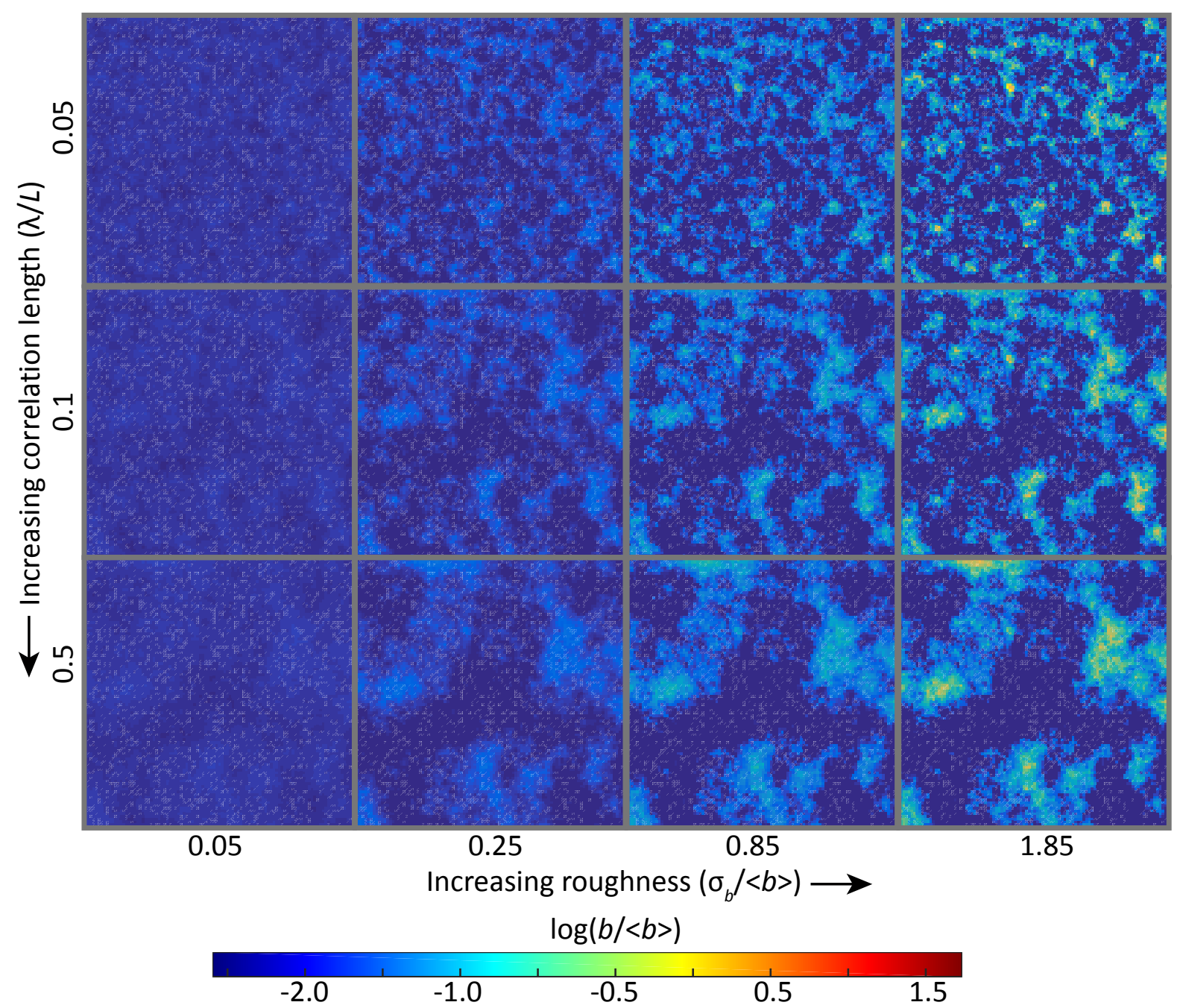

Figure 3. Examples of fractures with aperture field following log-normal distribution with different roughness $\left(\sigma_{b} /\langle b\rangle\right)$ and correlation length $(\lambda)$, where $b$ is apparent aperture with standard deviation $\sigma_{b}$ of aperture and arithmetic mean aperture $\langle b\rangle, L$ is the total length of fractures. 

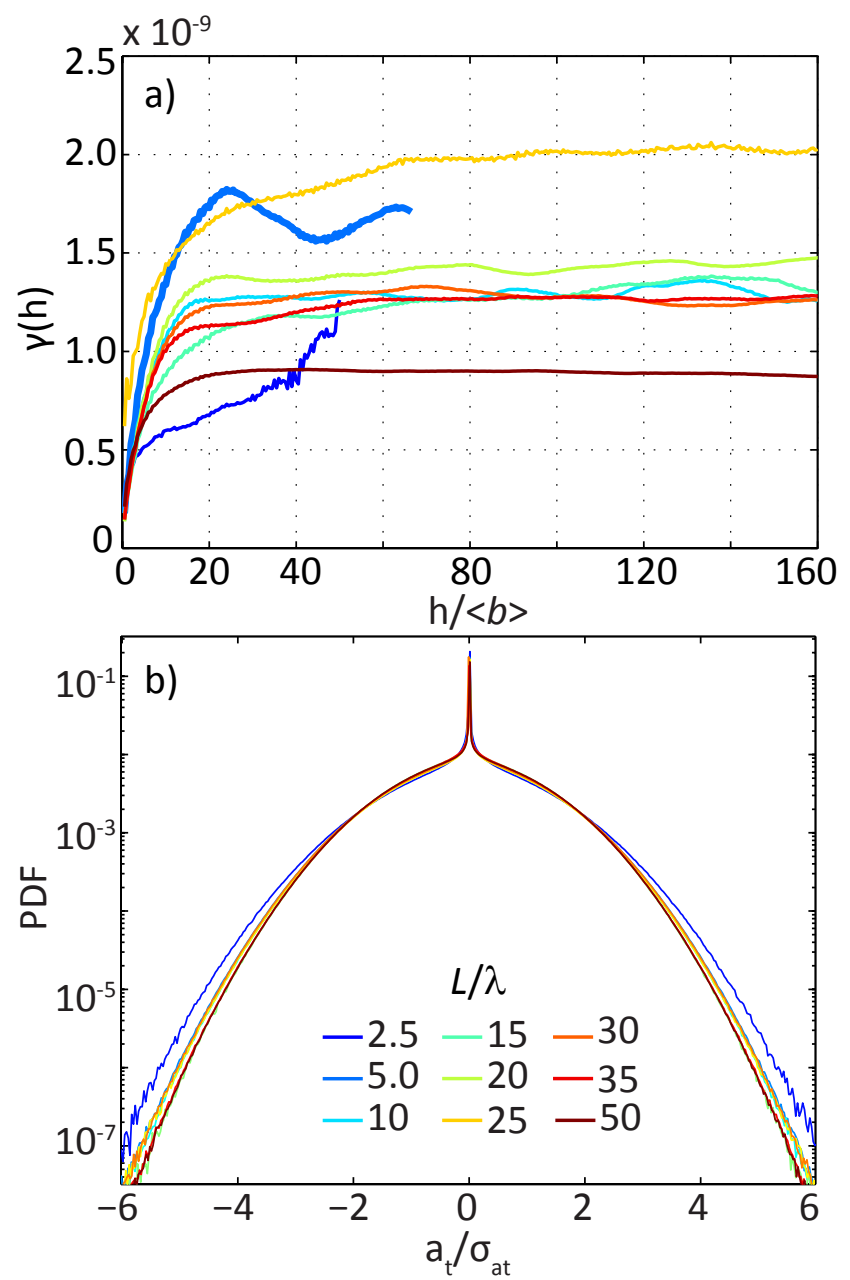

Figure 4. Statistical properties of the flow field for fractures with different length. (a) Experimental variogram $\gamma(\mathrm{h})$ for the longitudinal velocity field for the studied fractures; $h$ is lag distance, $\langle b\rangle$ is the arithmetic mean of aperture $b$. (b) Probability density function (PDF) of normalized Lagrangian acceleration of particles in the $x$ direction $\left(a_{t}(x)\right)$, where $a_{t}$ is acceleration with its standard deviation $\left(\sigma_{\mathrm{at}}\right), L$ is total length and $\lambda$ is the correlation length of aperture field. 

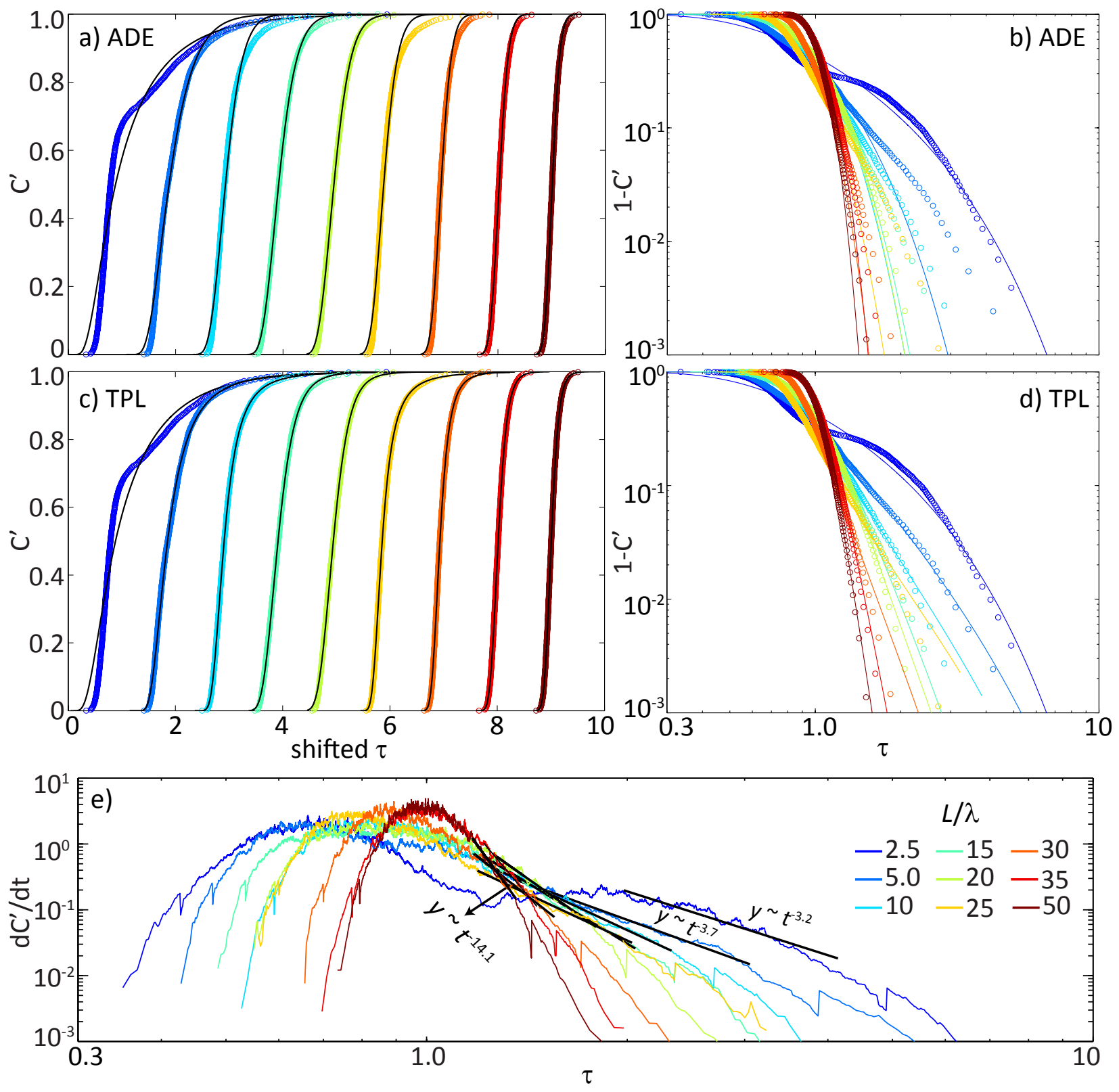

Figure 5. Breakthrough curves (BTCs) generated from particle tracking random walk (hollow circles), and inverse BTCs from solving the advection-dispersion equation (5a-5b, solid curves) and continuous random walk ( $5 c-5 d$, solid curves) for fractures with increasing scales (total length $L$ / correlation length $\lambda$ ). Each BTC group was shifted by 1 pore volume $\tau$ with each incremental increase in scale in $5 \mathrm{a}$ and $5 \mathrm{c}$. The corresponding residence time distributions (RTD) are shown in 5e with fitted power law shown as black lines. $C$ represents normalized concentration. 


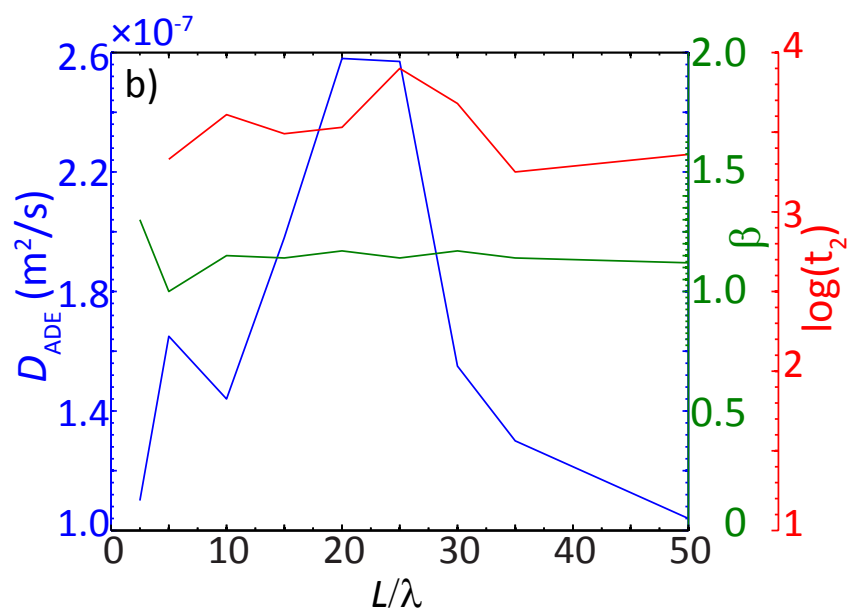

Figure 6 . The scale-(in)dependence of transport parameters: 1-D ADE dispersion coefficient $\left(D_{A D E}\right), C T R W-T P L \beta$, and CTRW-TPL truncation time $t_{2}$. 


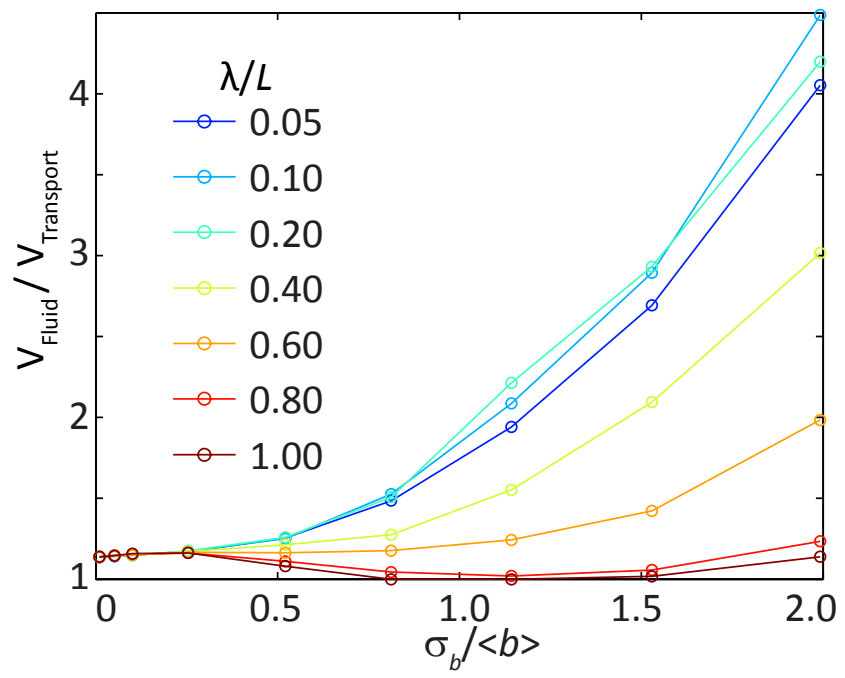

Figure 7. The ratio of fluid velocity $\left(\mathrm{V}_{\text {Fluid }}\right)$ to effective transport velocity $\left(\mathrm{V}_{\text {Transport }}\right)$ is a function of fracture roughness $\left(\sigma_{b} /\langle b\rangle\right)$ and aperture correlation length $(\lambda)$, where $V_{\text {Transport }}$ is obtained by fitting breakthrough curves to the advection-dispersion equation, $b$ is apparent aperture with standard deviation $\sigma_{b}$ of aperture and arithmetic mean aperture $\langle b\rangle, L$ is the total length of fracture. 


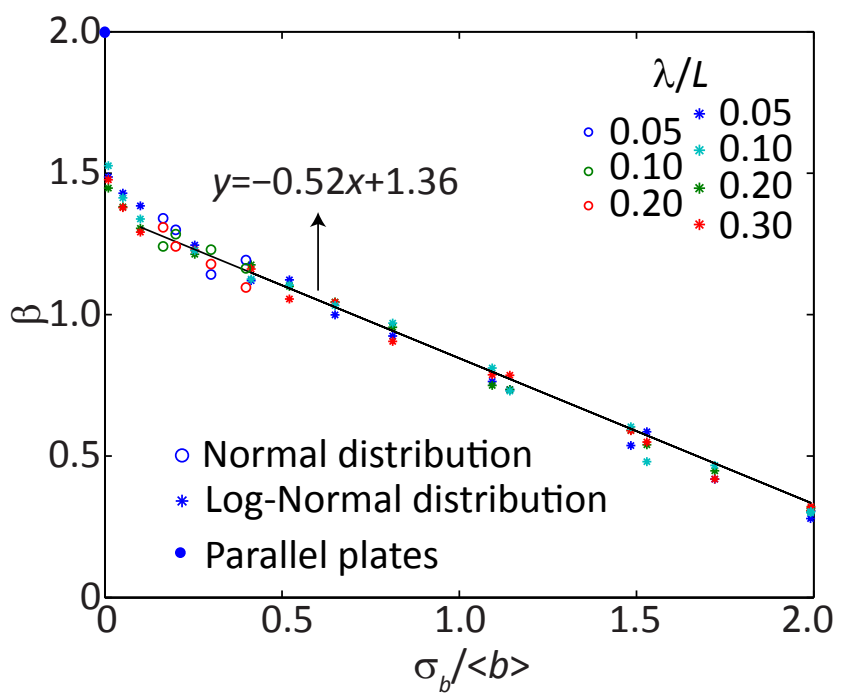

Figure 8. Dependence of parameter $\beta$ in the TPL model on fracture roughness $\left(\sigma_{b} /<b>\right)$, where $b$ is apparent aperture with standard deviation $\sigma_{b}$ of aperture and arithmetic mean aperture $\langle b\rangle, L$ is the total length of studied fracture. Color represents different aperture correlation length $(\lambda)$ following normal and log-normal distributions as shown by hollow circle and asterisk, respectively, filled blue circle refers to the ideal parallel plates model. The black line is the linear fit for $\beta$ with $\left.\sigma_{b} /<b\right\rangle$. 